\title{
Observing $\mathbf{H}_{2}$ Emission in Forming Galaxies
}

\author{
Kazuyuki Omukai ${ }^{1,2}$ and Tetsu Kitayama ${ }^{3}$ \\ omukai@astro.ox.ac.uk; kitayama@ph.sci.toho-u.ac.jp
}

\begin{abstract}
We study the $\mathrm{H}_{2}$ cooling emission of forming galaxies, and discuss their observability using the future infrared facility $S A F I R$. Forming galaxies with mass $\lesssim 10^{11} M_{\odot}$ emit most of their gravitational energy liberated by contraction in molecular hydrogen line radiation, although a large part of thermal energy at virialization is radiated away by the $\mathrm{H}$ Ly $\alpha$ emission. For more massive objects, the degree of heating due to dissipation of kinetic energy is so great that the temperature does not drop below $10^{4} \mathrm{~K}$ and the gravitational energy is emitted mainly by the Ly $\alpha$ emission. Therefore, the total $\mathrm{H}_{2}$ luminosity attains the peak value of $L_{\mathrm{H}_{2}} \sim 10^{42} \mathrm{ergs} / \mathrm{s}$ for forming galaxies whose total mass $M_{\text {tot }} \sim 10^{11} M_{\odot}$. If these sources are situated at redshift $z \sim 8$, they can be detected by rotational lines of $0-0 \mathrm{~S}(3)$ at $9.7 \mu \mathrm{m}$ and $0-0 \mathrm{~S}(1)$ at $17 \mu \mathrm{m}$ by $S A F I R$. An efficient way to find such $\mathrm{H}_{2}$ emitters is to look at the Ly $\alpha$ emitters, since the brightest $\mathrm{H}_{2}$ emitters are also luminous in the Ly $\alpha$ emission.
\end{abstract}

Subject headings: cosmology: theory — galaxies: formation — infrared: galaxies

\section{Introduction}

In the last decade, observational studies on primeval galaxies has made great progress. For example, deep surveys in the optical (Williams et al. 1996), near-infrared (Maihara et al. 2001) and mid-infrared (Taniguchi et al. 1997) wavelengths have revealed early evolution of galaxies.

\footnotetext{
${ }^{1}$ Department of Physics, Denys Wilkinson Building, Keble Road, Oxford, OX1 3RH, UK

${ }^{2}$ Division of Theoretical Astrophysics, National Astronomical Observatory, Mitaka, Tokyo 181-8588, Japan

${ }^{3}$ Department of Physics, Toho University, Funabashi, Chiba 274-8510, Japan
} 
Since galaxies in the earliest phase consist mostly of gas, knowledge on interstellar medium in forming galaxies is valuable for understanding galaxy formation process. Molecular line observation has been a powerful tool in diagnosing the physical conditions in local star-forming regions (e.g., Dyson \& Williams 1997). Recently, molecular line emission has also been found to emanate from high-redshift galaxies and the number of detected sources has been increasing rapidly (e.g., Ohta et al. 1996; Omont et al. 1996; Combes 1999). Among theoretical studies, Spaans \& Silk (1997) investigated CO line emission from protogalaxies. However, the detection of metal lines depends on the degree of metal enrichment and thus preceeding star formation is required.

Can we detect molecular lines in primordial pristine gas clouds? The possibility of detecting $\mathrm{H}_{2}$ line emission from forming galaxies was discussed as early as the firm establishment of the Big-Bang cosmology (Saslaw \& Zipoy 1967). Other pioneering work includes that by Izotov \& Kolesnik (1984) and Shchekinov \& Éntél' (1985), which treats the $\mathrm{H}_{2}$ emission from proto-clusters of galaxies, i.e, the so-called "Zel'dovich pancakes". More recently, Ciardi \& Ferrara (2001) investigated the $\mathrm{H}_{2}$ emission from the super-shells created in the intergalactic medium as a result of multiple SN explosions in PopIII objects. They concluded that about 10 per cent of the SN mechanical energy is carried away by the $\mathrm{H}_{2} 1-0 \mathrm{~S}(1)$ rovibrational line. Stimulated by the recent theoretical understanding of the first star-forming regions (e.g., Omukai \& Nishi 1998; Abel, Bryan, \& Norman 2002; Bromm, Coppi, \& Larson 2002; Omukai \& Palla 2001, 2003), research has been vigorously carried out on the radiation from individual star-forming cores. Flower \& Pineau des Forêts (2001) calculated $\mathrm{H}_{2}$ emission from a J-type shock expected to appear in the prestellar collapse. Kamaya \& Silk (2002) studied the $\mathrm{H}_{2}$ emission from a collapsing prestellar core using a simplified model, and pointed out that the $0-0 \mathrm{~S}(3)$ line is the strongest during the prestellar collapse phase. Ripamonti et al. (2002) arrived at similar conclusions by a more refined calculation. Shibai et al. (2001) studied the detectability of $\mathrm{H}_{2}$ absorption lines in high-redshift objects. All these studies, however, suggest that the prospect for direct detection of $\mathrm{H}_{2}$ emission from forming galaxies is rather dim, though not rejected, even with next generation facilities.

On the other hand, Ly $\alpha$ emitting galaxies in high-redshifts have been observed (e.g., Hu, Cowie, \& McMahon 1998; Steidel et al. 2000; Kodaira et al. 2003). Stimulated by these findings, some authors have investigated the Ly $\alpha$ cooling radiation from protogalaxies by semi-analytical modeling (Haiman, Spaans, \& Quataert 2000) or by numerical simulations (Fardal et al. 2001). They concluded that the greater part of the gravitational energy is indeed emitted by the $\mathrm{H} \mathrm{Ly} \alpha$ line emission. In their model, however, $\mathrm{H}_{2}$-line cooling is not included, and the temperature in protogalaxies thus remains above $10^{4} \mathrm{~K}$, below which atomic cooling is strongly suppressed. Although this is a fair assumption for the massive objects that they considered (see $\S 3$ below), for smaller objects with total mass $<10^{11} M_{\odot}$, 
sufficient $\mathrm{H}_{2}$ can be formed as a result of enhanced ionization degree by virialization shock and its delayed non-equilibrium recombination (Shapiro \& Kang 1986; Susa et al. 1998; Oh \& Haiman 2002). By this mechanism, the temperature drops further below $10^{4} \mathrm{~K}$ due to $\mathrm{H}_{2}$ line cooling. Therefore, the greater part of gravitational energy liberated by contraction will be available for the $\mathrm{H}_{2}$ cooling radiation. In this paper, we study the $\mathrm{H}_{2}$ cooling radiation from those forming galaxies and discuss the prospect for observing such sources. Our result is quite encouraging, suggesting that $\mathrm{H}_{2}$ emission is indeed observable by the future infrared satellite mission SAFIR from sources as high as $z=8$.

The organization of the rest of this paper is as follows: In $\S 2$, the method of calculation is described. In $\S 3$, the prediction for the $\mathrm{H}_{2}$ emission from forming galaxies and its observability is discussed. Finally in $\S 4$, a brief summary and discussions are presented. Throughout the paper, we adopt a $\Lambda$ CDM cosmology in accordance with the WMAP data (Spergel et al. 2003): $\left(\Omega_{\mathrm{M}}, \Omega_{\Lambda}, \Omega_{\mathrm{B}}, h, \sigma_{8}\right)=(0.3,0.7,0.05,0.7,0.9)$.

\section{Model}

\section{1. $\mathrm{H}_{2}$ emission from forming galaxies}

In this section, we describe our model for evaluating $\mathrm{H}_{2}$ line emission. We use a simplified evolution model where a forming protogalaxy is treated as a uniform cloud.

As the initial state, we take an instance of virialization. We use the analytic formula for the density at virialization

$$
\rho_{\text {vir }}=\rho_{\text {crit }} \Delta_{\mathrm{c}}
$$

where

$$
\Delta_{\mathrm{c}}=18 \pi^{2}+82 x-39 x^{2}, \quad x=-\frac{\Omega_{\Lambda}}{\Omega_{0}\left(1+z_{\mathrm{vir}}\right)^{3}+\Omega_{\Lambda}},
$$

which has been found to provide a good description of the numerical results (Bryan \& Norman 1998).

The critical density at $z=z_{\text {vir }}$ can be written as

$$
\rho_{\text {crit }}=\rho_{\text {crit }, 0}\left[\Omega_{0}\left(1+z_{\text {vir }}\right)^{3}+\Omega_{\Lambda}\right]
$$

where $\rho_{\text {crit }, 0}$ is the present-day critical density.

The radius and temperature of objects of total mass $M_{\text {tot }}$ in this instance are

$$
R_{\mathrm{vir}}=\left(M_{\mathrm{tot}} / \frac{4}{3} \pi \rho_{\mathrm{vir}}\right)^{1 / 3}
$$


and

$$
T_{\mathrm{vir}}=\frac{G \mu m_{\mathrm{H}}}{5 k_{\mathrm{B}} R_{\mathrm{vir}}} M_{\mathrm{tot}},
$$

respectively.

We first describe the dissipationless case and later describe the prescription for the dissipation of kinetic energy. For the sake of simplicity, the dark matter density is assumed to be constant after the virialization. The evolution of gas density is computed by solving the virial equation for a spherical object

$$
\frac{d^{2} R}{d t^{2}}=-\frac{G M}{R^{2}}\left(1-\frac{T}{T_{\mathrm{vir}}(R)}\right)
$$

where

$$
T_{\text {vir }}(R)=\frac{G \mu m_{\mathrm{H}}}{5 k_{\mathrm{B}} R} M(R),
$$

and $M(R)$ is the mass enclosed within a spherical shell of radius $R$.

The thermal evolution is computed by solving the energy equation

$$
\frac{d e}{d t}=-p \frac{d}{d t}\left(\frac{1}{\rho}\right)-\Lambda_{\text {rad }}-\Lambda_{\text {chem }},
$$

where $e$ is the specific thermal energy

$$
e=\frac{1}{\gamma_{\mathrm{ad}}-1} \frac{k_{\mathrm{B}} T}{\mu m_{\mathrm{H}}},
$$

and $\Lambda_{\text {rad }}$ and $\Lambda_{\text {chem }}$ are the radiative and chemical cooling rates per unit mass, respectively.

The radiative cooling rate includes $\mathrm{H}_{2}$ line emission, $\mathrm{HD}$ line emission, atomic emission by $\mathrm{H}$ and $\mathrm{He}$, and Compton cooling. The $\mathrm{H}_{2}$ line cooling is treated as in Omukai (2001), where the effect of CMB is taken into account and the ortho-para ratio of 3:1 is assumed. For the HD cooling, the cooling function by Galli \& Palla (1998) is used. The effect of CMB for HD cooling is treated approximately by replacing the cooling rate by $\mathrm{HD}$ line $\Lambda_{\mathrm{HD}}(T)$ by $\Lambda_{\mathrm{HD}}(T)-\Lambda_{\mathrm{HD}}\left(T_{\text {rad }}\right)$ (e.g., Tegmark et al. 1997). The atomic emission includes collisional excitation cooling, recombination cooling, and bremsstrahlung of $\mathrm{H}$ and He. The coefficients given by Anninos et al. (1999) are used.

The above formulation neglects dissipation of kinetic energy of contracting gas; after the initial thermal energy is carried away by radiation, essentially all the gravitational energy liberated by contraction contributes to accelerating the gas infall. If the gas, however, settles into a Kepler rotation disk, the kinetic energy is reduced by a factor of 2 compared to the freefalling sphere with the same radius, i.e. half of the kinetic energy must be dissipated away. 
Such dissipation may proceed through the thermalization of kinetic energy by turbulent motion induced by the non-spherical nature of the collapse. To mimic this effect, we add a dissipation term in equations of motion and of energy;

$$
\frac{d^{2} R}{d t^{2}}=-\frac{G M}{R^{2}}\left(1-\frac{T}{T_{\mathrm{vir}}(R)}\right)+D_{\mathrm{trb}}
$$

and

$$
\frac{d e}{d t}=-p \frac{d}{d t}\left(\frac{1}{\rho}\right)-\Lambda_{\mathrm{rad}}-\Lambda_{\text {chem }}+\Gamma_{\mathrm{trb}},
$$

where $D_{\text {trb }}$ and $\Gamma_{\text {trb }}$ are the drag and heating terms due to dissipation of kinetic energy. To compute these quantities, we assume that a fraction $f_{\text {trb }}$ of kinetic energy is thermalized during the collapse. Specifically, we first evaluate at each timestep the increment of kinetic energy in the dissipationless case $\Delta K_{0}$ using equations (6) and (8). We then solve equations (10) and (11) assuming that $f_{\operatorname{trb}} \Delta K_{0}=\Gamma_{\text {turb }} \Delta t$ is injected as heat and the actual increment of kinetic energy is $\Delta K=\left(1-f_{\text {trb }}\right) \Delta K_{0}$. In reality, the value of $f_{\text {trb }}$ is likely to lie between 0.5 (for the Kepler rotating disk) and 0 (for the spherical collapse). We adopt $f_{\text {trb }}=0.25$ as our fiducial value, while we also study the dissipationless case $\left(f_{\text {trb }}=0\right)$ for comparison.

The freeze-out values of chemical abundances are used for the initial abundances for the computation: $y_{e}=3 \times 10^{-4}, y_{H_{2}}=1 \times 10^{-6}$ and $y_{H D}=1 \times 10^{-9}$ (Galli \& Palla 1998). The chemical reactions between $\mathrm{H}$, and He compounds are solved following Omukai (2000; reactions 1-22), with HD-bearing reactions of D3, D4, D8, and D10 in Galli \& Palla (1998) added. We find, however, that HD cooling is not important in all cases we studied.

The cloud collapse is followed from the virialization until either the local Hubble time

$$
t_{\mathrm{H}}(z)=\frac{2}{3 H_{0}}\left(1-\Omega_{0}\right)^{-1 / 2} \sinh ^{-1}\left[\frac{\left(1-\Omega_{0}\right)^{1 / 2}}{\Omega_{0}^{1 / 2}(1+z)^{3 / 2}}\right]
$$

elapses or the radius reaches the rotation barrier

$$
R_{\text {rot }}=0.06\left(\frac{f_{\text {gas }}}{0.17}\right)^{-1}\left(\frac{\lambda_{\text {turn }}}{0.05}\right)^{2} R_{\text {vir }}
$$

where $f_{\text {gas }}$ is the mass fraction of gas. We adopt a median value 0.05 for the dimensionless spin parameter at turn-around $\lambda_{\text {turn }}$ (Barns \& Efstathiou 1987) and the gas density enhances by a factor of 5000 between $R_{\text {vir }}$ and $R_{\text {rot }}$. The duration of emission is therefore

$$
t_{\mathrm{em}}(M, z)=\min \left[t_{\mathrm{H}}(z), t_{\mathrm{rot}}(M, z)\right]
$$

where $t_{\text {rot }}(M, z)$ is the time taken to reach $R_{\text {rot }}$ from $R_{\text {vir }}$. 


\subsection{Merger probability of protogalaxies}

In predicting the luminosity and the abundance of protogalaxies, we also take into account explicitly hierarchical clustering and mergers of these objects. This is of particular importance at high redshift, say $z \gtrsim 8$, where a significant fraction of galaxy-scale halos merges into larger halos before the free-fall time is reached. For such halos, the duration of emission will be shorter than that estimated in Sec 2.1.

We incorporate the variations in the emission times by defining the "merger probability weighed average luminosity" of a protogalaxy with total mass $M$ at redshift $z$ as

$$
L_{\text {line }}(M, z)=\frac{\int_{t(z)-t_{\mathrm{em}}(M, z)}^{t(z)} L_{\text {line }}^{\mathrm{int}}\left(M, z, z_{f}\right) \frac{d p\left(M, z, z_{f}\right)}{d z_{f}}\left|\frac{d z_{f}}{d t_{f}}\right| d t_{f}}{\int_{t(z)-t_{\mathrm{em}}(M, z)}^{t(z)} \frac{d p\left(M, z, z_{f}\right)}{d z_{f}}\left|\frac{d z_{f}}{d t_{f}}\right| d t_{f}}
$$

where $L_{\text {line }}^{\text {int }}\left(M, z, z_{f}\right)$ is the intrinsic line luminosity at $z$ of the galaxy virialized at $z_{f}$, corresponding to the cosmic time $t_{f} \equiv t\left(z_{f}\right)$, and $d p\left(M, z, z_{f}\right) / d z_{f}$ is the probability distribution of formation epochs of virialized halos. The former quantity is computed using the model described in Sec 2.1. Since $t_{\mathrm{em}}(M, z)$ is typically an order of magnitude smaller than $t(z)$, we neglect the changes in $\rho_{\text {vir }}$ and $R_{\text {vir }}$ between $z$ and $z_{f}$. For the latter we adopt the prescription of Lacey \& Cole (1993) as described in Kitayama \& Suto (1996); the formation epoch $z_{f}$ is defined as the time at which a halo assembles at least half of its final mass. In the rest of this paper, unless otherwise stated, we simply refer as "luminosity" to that given in equation (15).

The number density of protogalaxies in accord with the above definition of average luminosity is

$$
\frac{d n\left(M, z ; L_{\text {line }}\right)}{d M}=\frac{d n_{\mathrm{PS}}(M, z)}{d M} \int_{t(z)-t_{\mathrm{em}}(M, z)}^{t(z)} \frac{d p\left(M, z, z_{f}\right)}{d z_{f}}\left|\frac{d z_{f}}{d t_{f}}\right| d t_{f},
$$

where $d n_{\mathrm{PS}}(M, z) / d M$ is the Press-Schechter (1974) mass function.

If strong bias is present in such high redshift we consider here, namely $z \sim 10-20$, the number of observable objects increases remarkably. In this sense, our result, which corresponds to the no bias case, can be regarded as the lower bound on the number of objects. 


\section{Results}

\section{1. $\mathbf{H}_{2}$ emission from individual objects}

In the following, we consider two epochs in the early universe, $z=20$ and 8 . The former corresponds to the typical epoch for the first star formation (e.g., Abel et al. 2002). The latter is believed to be shortly before the completion of the cosmic reionization from the observation of high-redshift quasars (e.g., Becker et al. 2001). Direct observation of objects forming in this epoch is therefore intersting for understanding the reionization process.

The total luminosity in $\mathrm{H}_{2}$ lines is shown in Figure 1 as a function of the total mass of the objects, along with the Ly $\alpha$ luminosity for the virial redshift (a) $z_{\text {vir }}=20$ and (b) $z_{\text {vir }}=8$. The corresponding virial temperature and the observed flux are indicated in the top and right axes. The observed flux in a line is calculated using the usual relation

$$
S_{\text {line }}=L_{\text {line }} / 4 \pi d_{L}(z)^{2}
$$

where $d_{L}(z)$ is the luminosity distance to the source at redshift $z$. The contribution to the total $\mathrm{H}_{2}$ luminosity from each line is shown in Figure 2 for the fiducial case $\left(f_{\text {trb }}=0.25\right)$ and in Figure 3 for the dissipationless case $\left(f_{\text {trb }}=0\right)$. Important lines are listed in Table 1 (for $z=20$ ) and 2 (for $z=8$ ) for the fiducial case.

We first focus on the fiducial case $f_{\text {trb }}=0.25$. From the $\mathrm{H}_{2}$ emission property, the protogalactic clouds can be categorized into the following four mass ranges. The temperature evolution for typical cases in these regimes is shown in Figure 4 for $z=20$.

- Pop III objects $\left(M_{\text {tot }} \lesssim 10^{8} M_{\odot}\right)$

Small objects whose virial temperature $T_{\text {vir }} \lesssim 10^{4} \mathrm{~K}$ cannot excite atomic hydrogen, and cool only by $\mathrm{H}_{2}$ lines. Thus both the thermal energy at virialization and the gravitational energy liberated by the collapse are converted to $\mathrm{H}_{2}$ emission. The dominant lines are the lowest excitation ones, e.g., 0-0S(0) at $28 \mu \mathrm{m}$ and $0-0 \mathrm{~S}(1)$ at $17 \mu \mathrm{m}$ (see Figure 2).

- dwarf galaxies $\left(10^{8} M_{\odot} \lesssim M_{\text {tot }} \lesssim 10^{11} M_{\odot}\right)$

Clouds with a virial temperature exceeding $10^{4} \mathrm{~K}$ can cool by Ly $\alpha$ radiation. As we can see in Figure 4, the temperature first drops rapidly from the virial temperature to that appropriate for $\mathrm{H}_{2}$ cooling $\left(\sim 10^{3} \mathrm{~K}\right)$. The initial thermal energy is radiated away by the atomic radiation processes including the Ly $\alpha$ emission $\left(T \gtrsim 10^{4} \mathrm{~K}\right)$ and by the $\mathrm{H}_{2}$ line emission $\left(T \lesssim 10^{4} \mathrm{~K}\right)$. The collapse proceeds quasi-isothermally thereafter due to the $\mathrm{H}_{2}$ line emission. This can be observed in Figure 1 as the emergence of 
Ly $\alpha$ luminosity. At the same time, the slope of the increase of the $\mathrm{H}_{2}$ line luminosity toward higher mass flattens slightly around $T_{\text {vir }} \simeq 10^{4} \mathrm{~K}$. The low excitation lines, e.g. 0-0S(1) at $17 \mu \mathrm{m}$ and $0-0 \mathrm{~S}(3)$ at $9.7 \mu \mathrm{m}$, are still dominant (Figure 2).

- giant galaxies $\left(10^{11} M_{\odot} \lesssim M_{\text {tot }} \lesssim 10^{12} M_{\odot}\right)$

Since the available gravitational energy increases toward massive objects, the heating rate by the dissipation of kinetic energy also increases. For objects $>10^{11} M_{\odot}$, the heating becomes so great that the temperature does not fall below $10^{4} \mathrm{~K}$. These objects collapse isothermally at $10^{4} \mathrm{~K}$, after initial rapid cooling (see Figure 4 a). During this isothermal collapse, a large fraction of energy is emitted in the Ly $\alpha$ radiation. Then the Ly $\alpha$ luminosity exceeds the $\mathrm{H}_{2}$ luminosity (Figure 1) for those objects. Despite the high temperature, a small amount of $\mathrm{H}_{2}$ is present. Only a small fraction of gravitational energy is emitted in the $\mathrm{H}_{2}$ lines. This can be observed as a plateau in the $\mathrm{H}_{2}$ luminosity in Figure 1 , as the larger mass compensates for the lower $\mathrm{H}_{2}$ emissivity. Corresponding to the abrupt change in thermal property, the dominant lines are altered (see Figure 2). Because the collapse proceeds with high temperature, high excitation lines become more important. The strongest line is $0-0 \mathrm{~S}(3)$ at $9.7 \mu \mathrm{m}$. Although $0-0 \mathrm{~S}(1)$ is still in the second place, the vibrational line $1-0 \mathrm{~S}(3)$ at $2.0 \mu \mathrm{m}$ also becomes important. These objects are the brightest ones in $\mathrm{H}_{2}$ emission and the $\mathrm{H}_{2}$ luminosity reaches about $3 \times 10^{41} \mathrm{ergs} \mathrm{s}^{-1}$. Note that these are also most luminous in Ly $\alpha$ emission.

- no-cooling objects $\left(M_{\text {tot }} \gtrsim 10^{12} M_{\odot}\right)$

For these massive objects, both $\mathrm{Ly} \alpha$ and $\mathrm{H}_{2}$ luminosities decline rapidly, because, in the redshift range we are considering, such massive objects cannot cool within the merging timescale and then remain in the virialization state (Silk 1977; Rees \& Ostriker 1977).

We stress the importance of the dissipation of kinetic energy. Early studies by Saslow \& Zipoy (1967) and by Izotov \& Kolesnik (1984) did not take the dissipation into account. As we can see in Figure 1, the maximum $\mathrm{H}_{2}$ luminosity would be smaller by an order of magnitude without the dissipation of kinematic energy. In this case, only the portion of the initial thermal energy below $10^{4} \mathrm{~K}$ would be available for $\mathrm{H}_{2}$ emission, with most gravitational energy being converted into kinetic energy. The discrepancy in emitted energy increases toward higher mass because of the larger gravitational energy to be released during the collapse; in the dissipationless case, the $\mathrm{H}_{2}$ luminosity increases only linearly in mass, while in the dissipational case it increases approximately as $\propto M_{\mathrm{tot}}^{1.5}$. 


\subsection{Number counts of $\mathrm{H}_{2}$ emitters and their contribution to the far-IR background light}

$\mathrm{H}_{2}$-line photons emitted at $z \simeq 10$ have been red-shifted to the far-infrared (FIR) wavelength today. The future space telescope, the Single Aperture Far-Infrared Observatory $^{4}(S A F I R)$, is planned to have unprecedented sensitivity at the FIR and submillimeter wavelengths, with the limiting flux of $\sim 10^{-19} \mathrm{erg} \mathrm{s}^{-1} \mathrm{~cm}^{-2}$ in FIR. With this limiting flux, brightest sources at $z=8$ can be observable by the $0-0 \mathrm{~S}(3) 9.7 \mu \mathrm{m}$ line (Figure $2 \mathrm{~b}$ ), while at redshift $z=20$, even the brightest $\mathrm{H}_{2}$ emitters are below this threshold (Figure 2 a).

Figure 5 shows the number of sources per unit solid angle above the limiting flux of (a) $10^{-19} \mathrm{erg} \mathrm{s}^{-1} \mathrm{~cm}^{-2}$ and (b) $10^{-20} \mathrm{erg} \mathrm{s}^{-1} \mathrm{~cm}^{-2}$ whose emission redshift is larger than $z$. For the limiting flux $10^{-19} \mathrm{erg} \mathrm{s}^{-1} \mathrm{~cm}^{-2}$, which is the planned limit for SAFIR, although the number of sources with their $\mathrm{H}_{2}$ total flux exceeding this limit is about $10^{6} \operatorname{str}^{-1}$ as early as $z=10$, even the strongest line 0-0S(3) $9.7 \mu \mathrm{m}$ cannot be observed until $z=8$ (see Figure 5 a). We can understand this from Figure 2 (b); at $z=8$, although the maximum total $\mathrm{H}_{2}$ flux exceeds $10^{-19} \mathrm{erg} \mathrm{s}^{-1} \mathrm{~cm}^{-2}$ by some factor, even the most dominant line 0-0S(3) $9.7 \mu \mathrm{m}$ barely reaches this limiting flux because of sharing the luminosity with other lines.

For the limiting flux $10^{-20} \mathrm{erg} \mathrm{s}^{-1} \mathrm{~cm}^{-2}$, the brightest objects at $z=20$ could be visible by the $0-0 \mathrm{~S}(3) 9.7 \mu \mathrm{m}$ line and the $0-0 \mathrm{~S}(1) 17 \mu \mathrm{m}$ line (Figure $2 \mathrm{a}$ ), although such massive objects are still very rare at $z=20$ (Figure $5 \mathrm{~b}$ ). For this limiting flux, the number of sources observable by these lines exceeds $\sim 100 \operatorname{str}^{-1}$ at $z \lesssim 15$.

Figure 6 further illustrates the number of $\mathrm{H}_{2}$ emitters for each line above the limiting flux per unit redshift range at $z=8$. At the limiting flux of $S A F I R, 10^{-19} \mathrm{erg} \mathrm{s}^{-1} \mathrm{~cm}^{-2}$, observable lines are 0-0S(3) $9.7 \mu \mathrm{m}$, and possibly 0-0S(1) $17 \mu \mathrm{m}$, although the latter slightly falls short of $10^{-19} \mathrm{erg} \mathrm{s}^{-1} \mathrm{~cm}^{-2}$. If the limiting flux were an order of magnitude deeper, some vibrational lines including 1-0Q(1) $2.4 \mu \mathrm{m}$ and 1-0S(1) $2.1 \mu \mathrm{m}$ could also be detected.

Finally, Figure 7 displays the contribution of these $\mathrm{H}_{2}$ emitters to the extragalactic FIR background radiation. Also plotted for reference is the FIR background spectrum detected by $C O B E / F I R A S$ (Fixsen et al. 1998). The $\mathrm{H}_{2}$ line emission from forming galaxies makes only a negligible contribution to the background light, being smaller than the COBE/FIRAS result by several orders of magnitude.

\footnotetext{
${ }^{4}$ http://safir.jpl.nasa.gov/index.asp
} 


\section{Summary and Discussion}

We have evaluated the amount of $\mathrm{H}_{2}$ line radiation from forming galaxies. The $\mathrm{H}_{2}$ line luminosity reaches the maximum value of about $3 \times 10^{41} \mathrm{erg} \mathrm{s}^{-1}$ for protogalaxies of total mass $M_{\text {tot }} \sim 10^{11} M_{\odot}$. Below this mass scale, all the gravitational energy is emitted by $\mathrm{H}_{2}$ line radiation, while above this scale, a large part of the energy is emitted by Ly $\alpha$ radiation. Such brightest objects at $z \sim 8$ are detectable by the next generation telescope SAFIR via the $0-0 \mathrm{~S}(3) 9.7 \mu \mathrm{m}$ line emission and marginally detectable also via the $0-0 \mathrm{~S}(1) 17 \mu \mathrm{m}$ line. The cosmic FIR background radiation by the $\mathrm{H}_{2}$ emission is several orders of magnitude below the detected value of $C O B E / F I R A S$, and can be safely neglected.

The $\mathrm{H}_{2}$ luminosity from a protogalactic cloud has also been studied by Shchekinov (1991). Assuming that a protogalactic cloud consists of subclumps and that the luminosity is given by the sum of luminosity from each subclumps, he evaluated $L_{\mathrm{H}_{2}} \gtrsim 10^{42} \mathrm{erg} / \mathrm{sec}$ from a protogalactic cloud with gas mass $10^{11} M_{\odot}$ corresponding to total mass $M_{\text {tot }} \simeq 10^{12} M_{\odot}$. On the other hand, in our model the large protogalaxies of $M_{\text {tot }} \gtrsim 10^{12} M_{\odot}$ has no time to cool and then emit only weak $\mathrm{H}_{2}$ radiation (total $\mathrm{H}_{2}$ luminosty $L_{\mathrm{H}_{2}} \sim 10^{40} \mathrm{erg} / \mathrm{sec}$ ). This is because such inhomogenous clouds are regarded as a cluster of protogalactic clouds rather than a single cloud in our scheme. In fact, the sum of luminosity from a cluster with total mass of $M_{\text {tot }} \simeq 10^{12} M_{\odot}$ gives a similar value as that of Shchekinov (1991). For example, the luminosity of a protogalactic cloud of $M_{\mathrm{tot}}=10^{8} M_{\odot}$ collapsing at $z=20$ is $L_{\mathrm{H}_{2}} \sim 10^{8} \mathrm{erg} / \mathrm{sec}$ (Fig. 2 a). Then the $\mathrm{H}_{2}$ luminosity of a cluster consisting of $10^{4}$ such clouds is $\sim 10^{12} \mathrm{erg} / \mathrm{sec}$. Note that in order for this value to be the actual luminosity from the cluster, the collapse of the subclumps must be simultaneous. Since this is probably a rare occurence, this possibility is not considered in this paper.

As we mentioned in $\S 3$, the brightest $\mathrm{H}_{2}$ sources, "giant galaxies", are even more luminous in the Ly $\alpha$ emission. This has been pointed out also by Shchekinov (1991). who investigated pancaking shock of primordial clouds. Consequently, the most efficient way of finding $\mathrm{H}_{2}$ emitters is to look at the already detected Ly $\alpha$ emitters, rather than looking for them in a blank field. The origin of Ly $\alpha$ emitters found at high-redshift is still in dispute. If

Ly $\alpha$ emitters are originating in the cooling radiation, they might be bright as well as in the $\mathrm{H}_{2}$ emission. On the other hand, the Ly $\alpha$ emission might be originating in the recombination radiation due to stellar ionization (Partridge \& Peebles 1967). In this case, there is no reason to expect bright $\mathrm{H}_{2}$ emission from those sources. Nonetheless, since $\mathrm{H}_{2}$ is vulnerable to photodissociation by stars as we will discuss below, even if the $\mathrm{H}_{2}$ emission will not be detected, this will not demonstrate the recombination origin for the Ly $\alpha$ radiation.

Here, we discuss some effects that can alter the amount of the $\mathrm{H}_{2}$ luminosity from forming galaxies. The density contrast inside the clouds, in particular higher density than 
average in the inner region, results in luminosity higher by some factor, reducing the collapse timescale compared to the merging timescale. On the other hand, the following effects reduce the $\mathrm{H}_{2}$ luminosity: First, molecular hydrogen is vulnerable to photodissociation by stars. According to the recent WMAP results, the reionization epoch of the universe could be as early as $z \simeq 17 \pm 5$ (Spergel et al. 2003). At least below this redshift, protogalaxies are irradiated by the UV background radiation. According to our study, objects most luminous in $\mathrm{H}_{2}$ emission are massive ones whose mass is about $10 \%$ of $L_{*}$ galaxies today. Such massive objects are not vulnerable to photodissociation by UV background radiation. Therefore, our results remain unchanged except for "PopIII objects", for which the feedback effect is significant (Haiman, Rees, \& Loeb 1997; Omukai \& Nishi 1999; Ciardi et al. 2000; Kitayama et al. 2001; Ricotti et al. 2002). Although "giant galaxies" are not affected by the UV background radiation, an internal UV field created by local star formation might photodissociate molecular hydrogen and then reduce the amount of the $\mathrm{H}_{2}$ emission. Another possibility for reducing the $\mathrm{H}_{2}$ emissivity is by metal enrichment; with metals, the energy budget for $\mathrm{H}_{2}$ emission is reduced by the cooling via metal fine-structure and metastable lines (e.g., Dalgarno \& McCray 1972). For the metallicity $Z>10^{-2} Z_{\odot}$, the metal line cooling dominates over the $\mathrm{H}_{2}$ line cooling (e.g., Fall \& Rees 1985). Below this metallicity, the $\mathrm{H}_{2}$ cooling dominates and thus the effect of metal lines in reducing the $\mathrm{H}_{2}$ luminosity can be neglected. Further study of the influence of stellar feedback and metal enrichment on the $\mathrm{H}_{2}$ emission will be interesting.

We are grateful for helpful discussions with H. Matsuo, H. Susa, and M. Umemura. This work is supported in part by the Research Fellowship of the Japan Society for the Promotion of Science for Young Scientists (6819, KO), and the Grants-in-Aid by the Ministry of Education, Science and Culture of Japan (14740133, TK). 


\section{REFERENCES}

Abel, T., Bryan, G. L., \& Norman, M. L. 2002, Science, 295, 93

Anninos, P., Zhang, Y., Abel, T., Norman, M. L. 1997, NewA, 2, 209

Becker, R. H. et al. 2001, AJ, 122, 2850

Bromm, V., Coppi, P. S., \& Larson, R. B. 2002, ApJ, 564, 23

Bryan, G. L., \& Norman, M. L. 1998, ApJ, 495, 80

Ciardi, B., \& Ferrara, A. 2001, MNRAS, 324, 648

Ciardi, B., \& Ferrara, A., Governato, F., \& Jenkins, A. 2000, MNRAS, 318, 1068

Combes, F. 1999, in Proceedings of the ESO Workshop, Chemical Evolution from Zero to High Redshift, ed. J. R. Walsh, M. R. Rosa (Berlin: Splinger), 213

Dyson, J. E., \& Williams, D. A. 1997, The Physics of the Interstellar Medium, 2nd edition (Bristol:IOP Publishing)

Fall, S. M., \& Rees, M. J. 1985, ApJ, 298, 18

Fardal, M. A. et al. 2001, ApJ, 562, 605

Flower, D. R., \& Pineau des Forêts, G. 2001, MNRAS, 323, 672

Fixsen, D. J., Dwek, E., Mather, J. C., Bennett, C. L., Shafer, R. A., ApJ, 508, 123

Galli, D., \& Palla, F. 1998, A\&A, 335, 403

Haiman, Z., Rees, M. J., Loeb, A. 1997, ApJ, 476, 458

Haiman, Z., Spaans, M., \& Quataert, E. 2000, ApJ, 537, L5

Hu, E. M., Cowie, L. L., \& McMahon, R. G. 1998, ApJ, 502, L99

Izotov, Yu. I., \& Kolesnik, I. G. 1984, Soviet Astron., 28, 15

Kamaya, H., \& Silk, J. 2002, MNRAS, 332, 251

Kitayama, T., Susa, H. Umemura, M., \& Ikeuchi, 2001, MNRAS, 326, 1353

Kitayama, T., \& Suto, Y., 1996, ApJ, 469, 480

Kodaira, K., et al., 2003, PASJ, 55, L17 
Lacey, C. G., \& Cole, S., 1993, MNRAS, 262, 627

Maihara, T., et al. 2001, PASJ, 53, 25

Oh, S. P. \& Haiman, Z. 2002, ApJ, 569, 558

Ohta, K., et al. 1996, Nature, 382, 426

Omont, A., et al. 1996, Nature, 382, 428

Omukai, K. 2000, ApJ, 534, 809

Omukai, K. 2001, ApJ, 546, 635

Omukai, K. \& Nishi, R. 1998, ApJ, 508, 141

Omukai, K. \& Nishi, R. 1999, ApJ, 518, 64

Omukai, K. \& Palla, F. 2001 ApJ, 561, L55

Omukai, K. \& Palla, F. 2003 ApJ, in press

Partridge, R. B., \& Peebles, P. J. E. 1967, ApJ, 147, 868

Press, W. H., \& Schechter, P., 1974, ApJ, 187, 425

Rees, M. J., \& Ostriker, J. 1977, MNRAS, 179, 541

Ricotti, M., Gnedin, N. Y., \& Shull, J. M. 2002, ApJ, 575, 49

Ripamonti, E., Haardt, F., Ferrara, A., \& Colpi, M. 2002, MNRAS, 334, 401

Saslaw, W. C., \& Zipoy, D. 1967, Nature, 216, 976

Shchekinov, Yu. 1991, Ap\&SS, 175, 57

Shchekinov, Yu. A., \& Éntél', M. B. 1985, Soviet Astron., 29, 491

Shibai, H., Takeuchi, T. T., Rengarajan, T. N., \& Hirashita, H. 2001, PASJ, 53, 589

Silk, J. 1977, ApJ, 211, 638

Spaans, M., \& Silk, J. 1997, ApJ, 488, L79

Spergel, D., et al. 2003, ApJ, submitted (astro-ph/0302209)

Steidel, C. C., et al. 2000, ApJ, 532, 170 
Susa, H., Uehara, H., Nishi, R., \& Yamada, M. 1998, Prog. Theor. Phys., 100, 63

Taniguchi, Y., et al. 1997, A\&A, 328, L9

Williams, R. E., et al. 1996, AJ, 112, 1335 


\section{Figures}
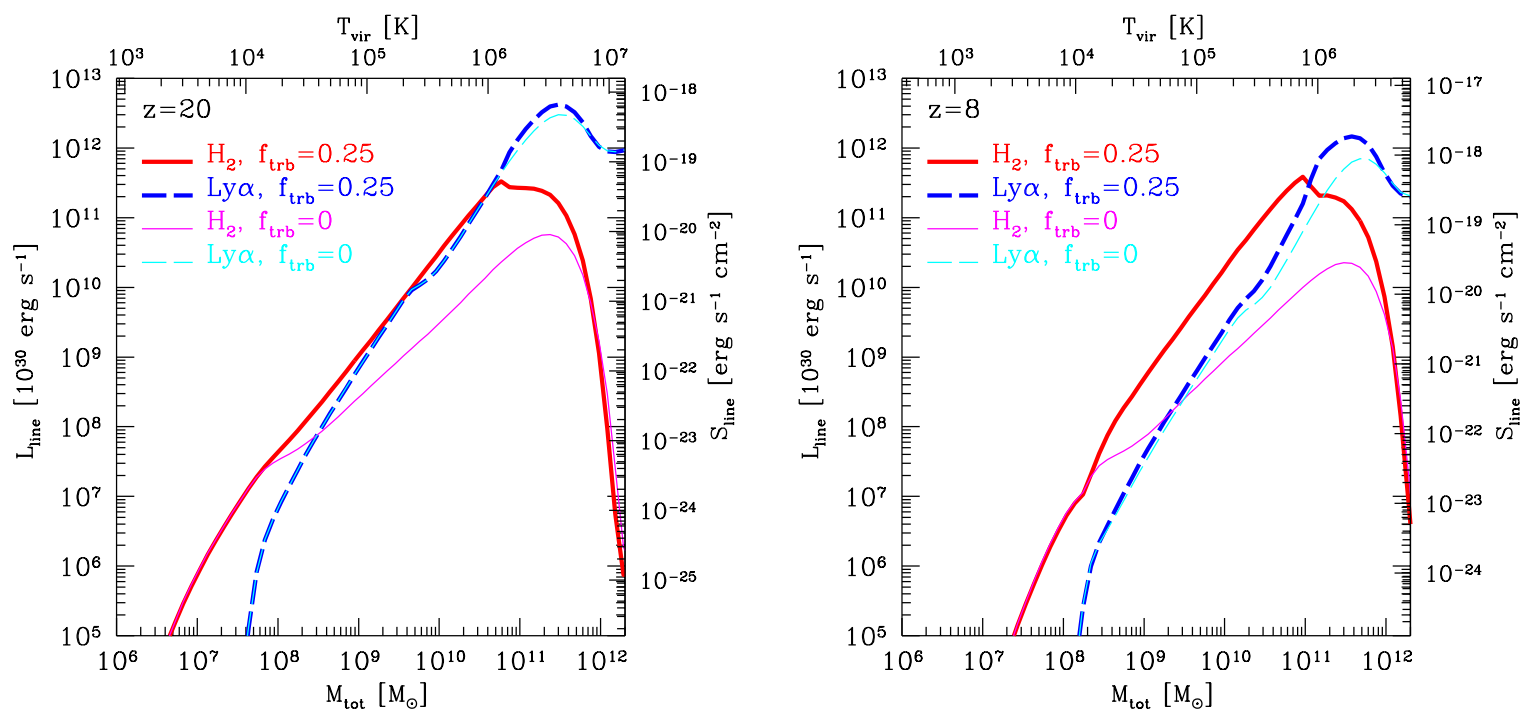

Fig. 1. - The total luminosity in $\mathrm{H}_{2}$ lines and the Ly $\alpha$ line luminosity as a function of the mass of protogalactic clouds that collapses at (a) $z=20$, and (b) $z=8$. The luminosity is averaged over the merger probability of halos as in equation (15). The corresponding virial temperature is also shown along the top axis. The observed flux today is shown along the right axis. Both the fiducial case $\left(f_{\text {trb }}=0.25\right)$ and the dissipationless case $\left(f_{\text {trb }}=0\right)$ are shown.
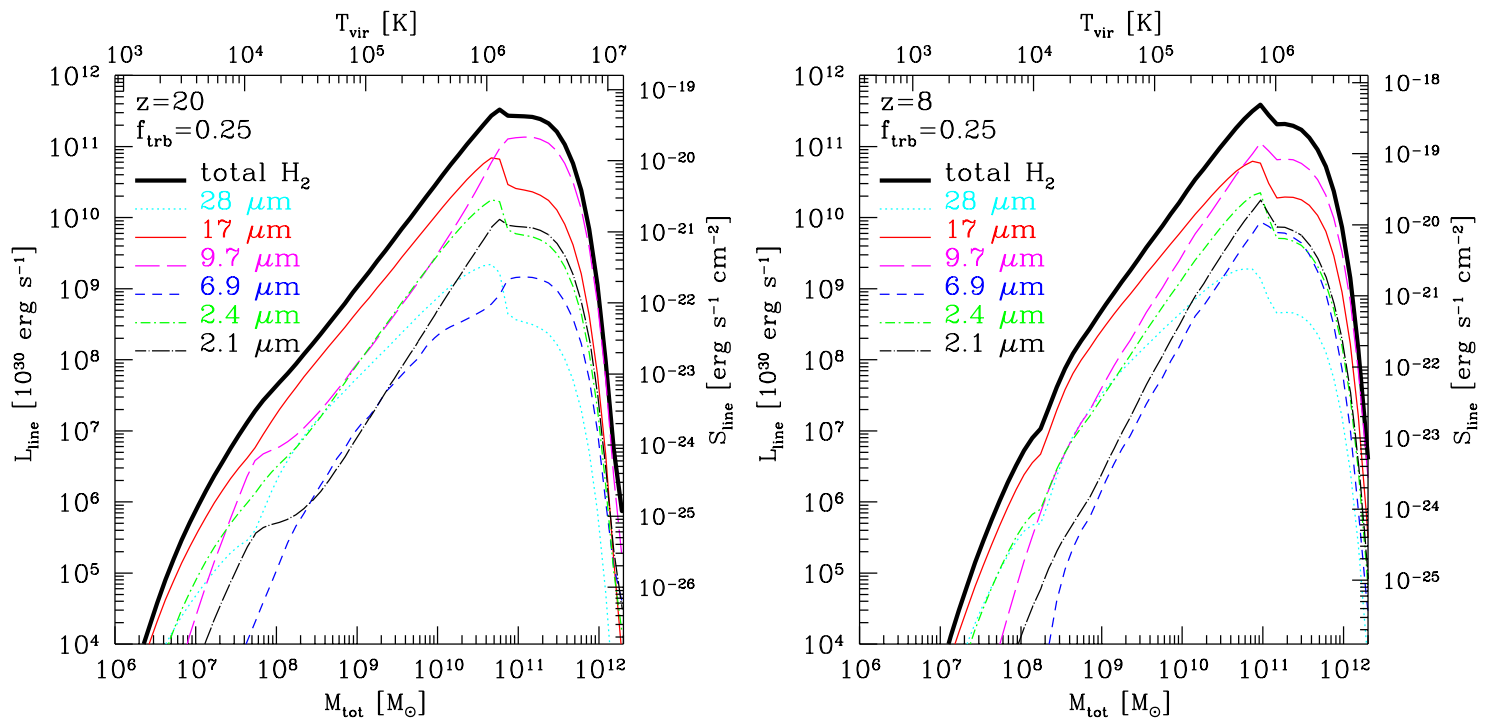

Fig. 2.- The contribution from individual lines to the $\mathrm{H}_{2}$ line luminosity in the fiducial case $\left(f_{\text {trb }}=0.25\right)$ for the collapse redshift of $(\mathrm{a}) \mathrm{z}=20$ and $(\mathrm{b}) \mathrm{z}=8$. The thick solid line shows 
the sum of all lines.
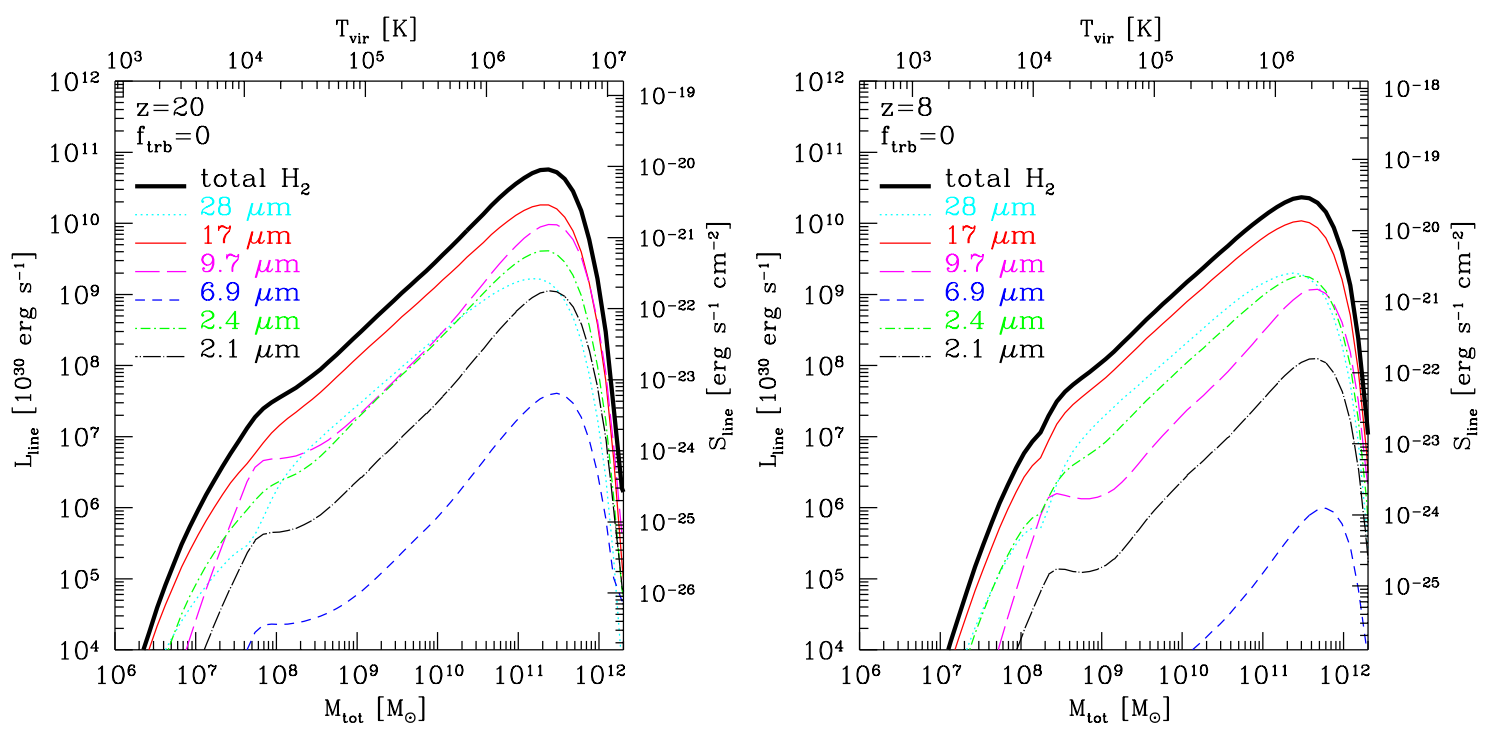

Fig. 3.- The same as Figure 2 but for the dissipationless case $\left(f_{\operatorname{trb}}=0\right)$.
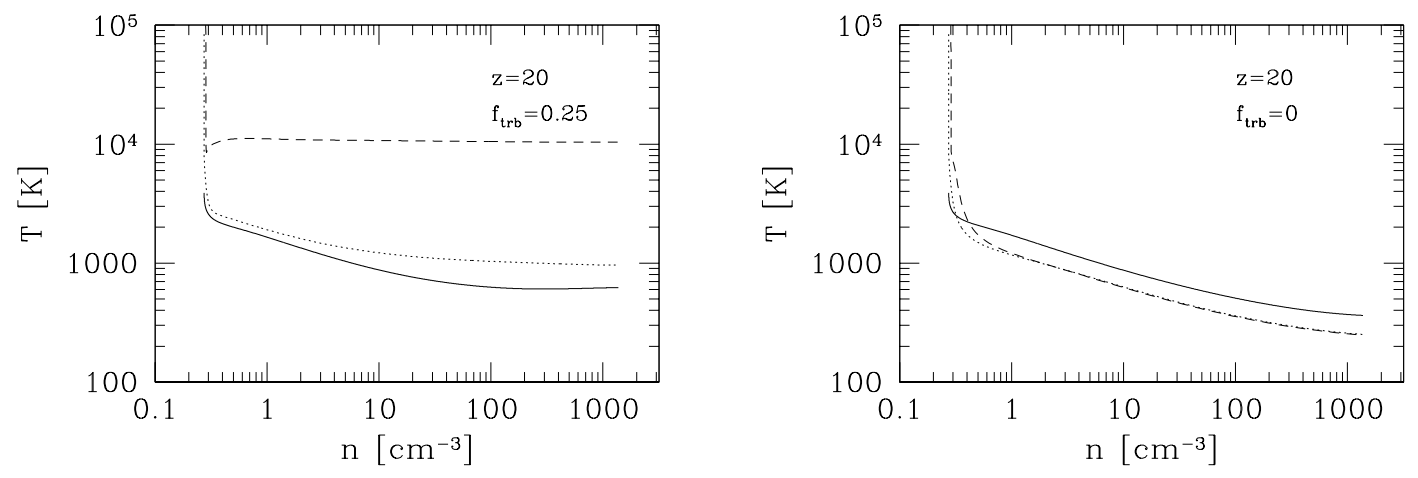

Fig. 4. - The temperature evolution of protogalaxies virializing at $z=20$ as a function of the $\mathrm{H}$ number density $n$ for (a)the fiducial case $\left(f_{\text {trb }}=0.25\right)$, and (b)the dissipationless case $\left(f_{\mathrm{trb}}=0\right)$. Illustrated are the protogalaxies of $M_{\mathrm{tot}}=10^{7}$ (solid lines), $10^{9}$ (dotted lines), and $10^{11} M_{\text {sun }}$ (dashed lines). 

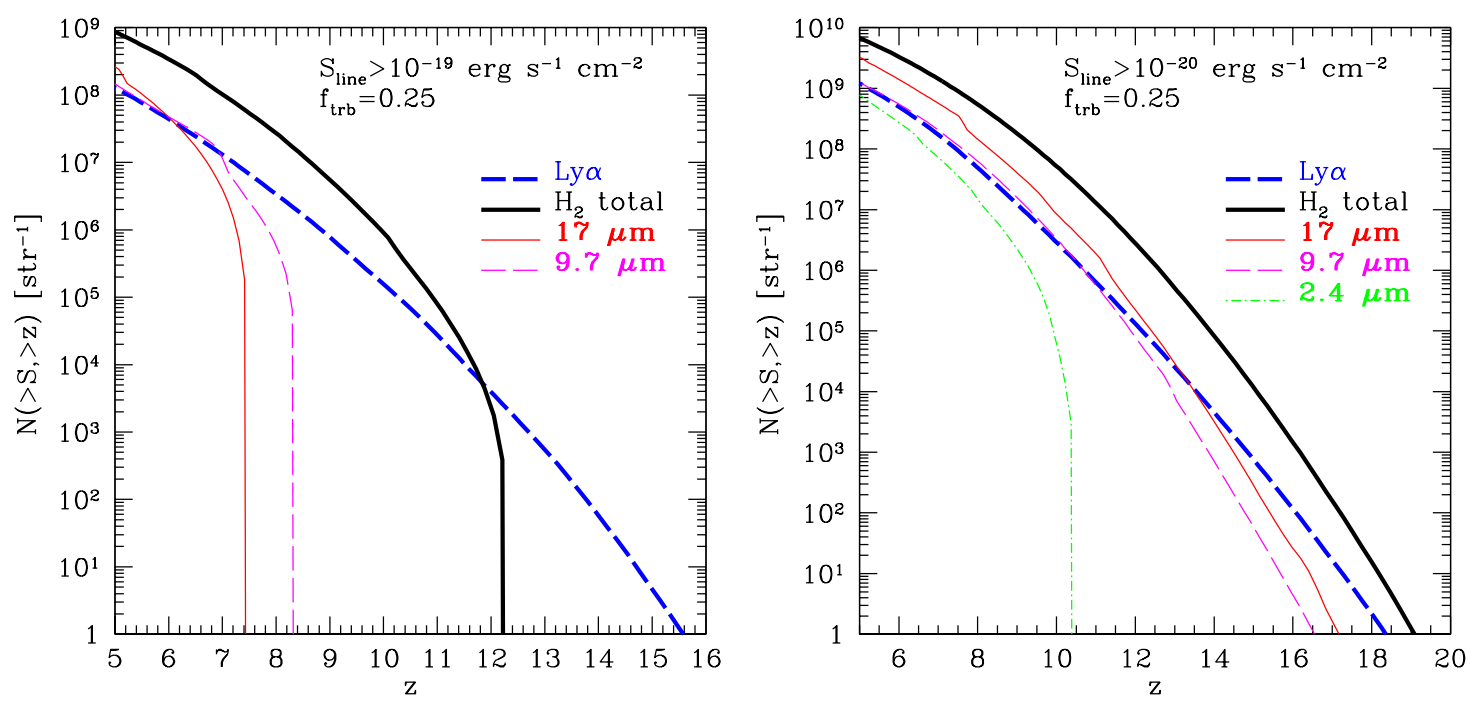

Fig. 5. - The number of sources below a redshift $z$ exceeding the limiting flux (a) $S_{\text {line }}>$ $10^{-19}\left(\mathrm{erg} \mathrm{s}^{-1} \mathrm{~cm}^{-2}\right)$, or (b) $S_{\text {line }}>10^{-20}\left(\mathrm{erg} \mathrm{s}^{-1} \mathrm{~cm}^{-2}\right)$. The thick solid line shows the total flux of $\mathrm{H}_{2}$ lines, and the thick dashed line, the Ly $\alpha$ flux. The flux of individual $\mathrm{H}_{2}$ lines $17 \mu \mathrm{m}$ and $9.7 \mu \mathrm{m}$ are also shown in thin lines.

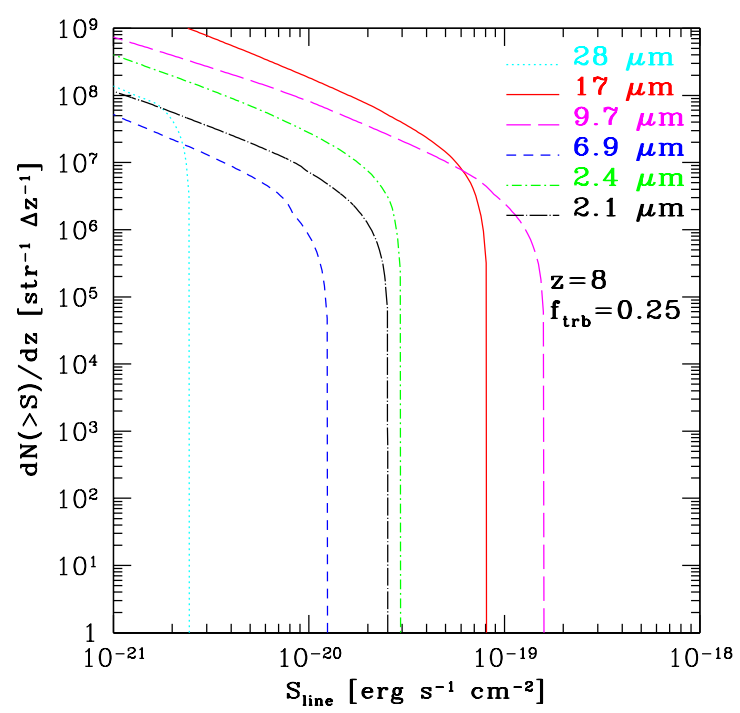

Fig. 6.- The number count of $\mathrm{H}_{2}$ emitting sources as a function of the minimum observed flux $S_{\text {line }}$ per unit redshift interval at $z=8$. 


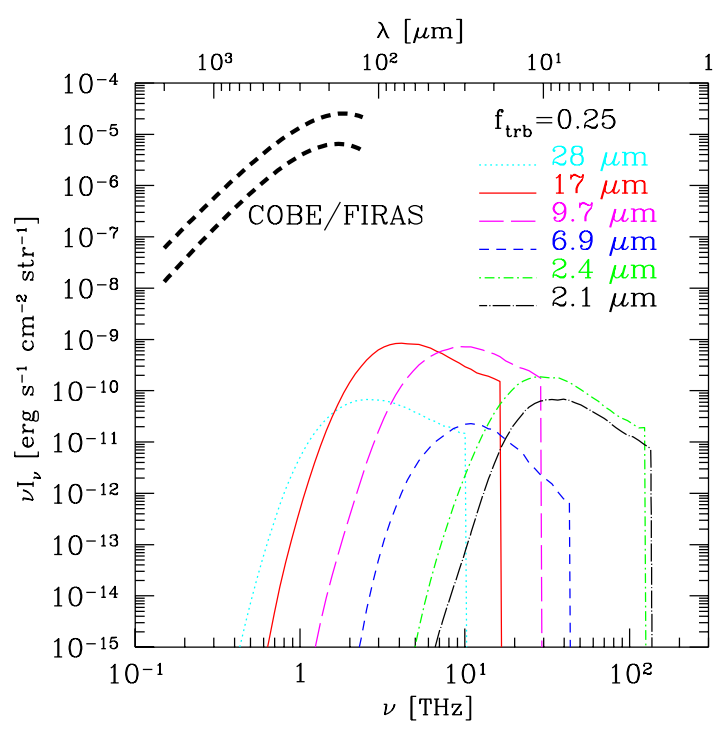

Fig. 7.- The contribution of $\mathrm{H}_{2}$ cooling radiation to the cosmic background in the fiducial case $\left(f_{\text {trb }}=0.25\right)$. Also shown is the IR background detected by COBE/FIRAS. 


\begin{tabular}{|c|c|c|c|c|c|c|c|}
\hline \multirow[t]{2}{*}{ line } & \multirow{2}{*}{$\begin{array}{c}\text { wavelength } \\
(\mu \mathrm{m})\end{array}$} & \multirow{2}{*}{$\begin{array}{r}\text { luminosity } \\
10^{7} M_{\odot} \\
\end{array}$} & \multirow{2}{*}{$\begin{array}{l}(\mathrm{erg} / \mathrm{s}) \\
10^{9} M_{\odot} \\
\end{array}$} & \multirow{2}{*}{\multicolumn{2}{|c|}{ observed flux }} & \multicolumn{2}{|l|}{$\left(\mathrm{erg} / \mathrm{cm}^{2} / \mathrm{s}\right)$} \\
\hline & & & & $10^{11} M_{\odot}$ & & $10^{9} M_{\odot}$ & $10^{11} M_{\odot}$ \\
\hline $0-0 \mathrm{~S}(0)$ & 28.3 & $4.83 \mathrm{E}+34$ & $5.49 \mathrm{E}+37$ & $3.54 \mathrm{E}+38$ & $7.94 \mathrm{E}-27$ & $9.02 \mathrm{E}-24$ & $\overline{5.81 \mathrm{E}-23}$ \\
\hline $0-0 \mathrm{~S}(1)$ & 17.1 & $3.42 \mathrm{E}+35$ & $4.67 \mathrm{E}+38$ & $2.50 \mathrm{E}+40$ & $5.61 \mathrm{E}-26$ & $7.66 \mathrm{E}-23$ & $4.11 \mathrm{E}-21$ \\
\hline $0-0 \mathrm{~S}(2)$ & 12.3 & $8.04 \mathrm{E}+34$ & $1.27 \mathrm{E}+38$ & $3.57 \mathrm{E}+40$ & $1.32 \mathrm{E}-26$ & $2.08 \mathrm{E}-23$ & $5.86 \mathrm{E}-21$ \\
\hline $0-0 \mathrm{~S}(3)$ & 9.69 & $2.48 \mathrm{E}+34$ & $8.78 \mathrm{E}+37$ & $1.34 \mathrm{E}+41$ & 4.07E-27 & $1.44 \mathrm{E}-23$ & $2.20 \mathrm{E}-20$ \\
\hline $0-0 \mathrm{~S}(5)$ & 6.95 & $3.52 \mathrm{E}+31$ & $1.11 \mathrm{E}+37$ & $1.46 \mathrm{E}+39$ & $5.78 \mathrm{E}-30$ & $1.82 \mathrm{E}-24$ & $2.39 \mathrm{E}-22$ \\
\hline $1-0 \mathrm{O}(3)$ & 2.81 & $6.85 \mathrm{E}+34$ & $7.26 \mathrm{E}+37$ & $4.98 \mathrm{E}+39$ & $1.13 \mathrm{E}-26$ & $1.19 \mathrm{E}-23$ & $8.18 \mathrm{E}-22$ \\
\hline $2-1 \mathrm{Q}(1)$ & 2.55 & $1.33 \mathrm{E}+34$ & $1.04 \mathrm{E}+37$ & $2.48 \mathrm{E}+39$ & $2.18 \mathrm{E}-27$ & $1.72 \mathrm{E}-24$ & 4.07E-22 \\
\hline $1-0 Q(1)$ & 2.41 & $7.99 \mathrm{E}+34$ & $8.47 \mathrm{E}+37$ & $5.81 \mathrm{E}+39$ & $1.31 \mathrm{E}-26$ & $1.39 \mathrm{E}-23$ & $9.54 \mathrm{E}-22$ \\
\hline $1-0 \mathrm{~S}(0)$ & 2.23 & $9.64 \mathrm{E}+33$ & $1.12 \mathrm{E}+37$ & $1.84 \mathrm{E}+39$ & $1.58 \mathrm{E}-27$ & $1.84 \mathrm{E}-24$ & $3.03 \mathrm{E}-22$ \\
\hline $1-0 \mathrm{~S}(1)$ & 2.12 & $4.49 \mathrm{E}+33$ & $8.27 \mathrm{E}+36$ & $7.37 \mathrm{E}+39$ & 7.37E-28 & $1.36 \mathrm{E}-24$ & $1.21 \mathrm{E}-21$ \\
\hline $1-0 \mathrm{~S}(3)$ & 1.96 & $1.73 \mathrm{E}+31$ & $2.83 \mathrm{E}+35$ & $1.86 \mathrm{E}+39$ & $2.84 \mathrm{E}-30$ & $4.64 \mathrm{E}-26$ & $3.06 \mathrm{E}-22$ \\
\hline total $\mathrm{H}_{2}$ & & $7.63 \mathrm{E}+35$ & $1.05 \mathrm{E}+39$ & $2.66 \mathrm{E}+41$ & $1.25 \mathrm{E}-25$ & $1.72 \mathrm{E}-22$ & $4.37 \mathrm{E}-20$ \\
\hline H Ly $\alpha$ & & $2.58 \mathrm{E}+26$ & $6.95 \mathrm{E}+38$ & $1.48 \mathrm{E}+42$ & $4.23 \mathrm{E}-35$ & $1.14 \mathrm{E}-22$ & $2.43 \mathrm{E}-19$ \\
\hline
\end{tabular}

Table 1: The $\mathrm{H}_{2}$ luminosity and observed flux from forming galaxies of total mass $10^{7} M_{\odot}, 10^{9} M_{\odot}$ and $10^{11} M_{\odot}$ at $z=20$. The quantities of $\mathrm{H}$ Ly $\alpha$ are also presented. 


\begin{tabular}{lcrllrll}
\hline line & $\begin{array}{r}\text { wavelength } \\
(\mu \mathrm{m})\end{array}$ & $\begin{array}{r}\text { luminosity } \\
10^{7} M_{\odot}\end{array}$ & $\begin{array}{l}(\mathrm{erg} / \mathrm{s}) \\
10^{9} M_{\odot}\end{array}$ & $10^{11} M_{\odot}$ & $\begin{array}{c}\text { observed flux } \\
\left(\mathrm{erg} / \mathrm{cm}^{2} / \mathrm{s}\right)\end{array}$ \\
\hline \hline $0-0 \mathrm{~S}(0)$ & 28.3 & $9.34 \mathrm{E}+32$ & $3.34 \mathrm{E}+37$ & $1.27 \mathrm{E}+39$ & $1.21 \mathrm{E}-27$ & $4.31 \mathrm{E}-23$ & $1.64 \mathrm{E}-21$ \\
$0-0 \mathrm{~S}(1)$ & 17.1 & $2.36 \mathrm{E}+33$ & $2.57 \mathrm{E}+38$ & $5.76 \mathrm{E}+40$ & $3.04 \mathrm{E}-27$ & $3.31 \mathrm{E}-22$ & $7.44 \mathrm{E}-20$ \\
$0-0 \mathrm{~S}(2)$ & 12.3 & $2.33 \mathrm{E}+31$ & $6.02 \mathrm{E}+37$ & $5.05 \mathrm{E}+40$ & $3.01 \mathrm{E}-29$ & $7.77 \mathrm{E}-23$ & $6.51 \mathrm{E}-20$ \\
$0-0 \mathrm{~S}(3)$ & 9.69 & $2.02 \mathrm{E}+30$ & $3.99 \mathrm{E}+37$ & $1.25 \mathrm{E}+41$ & $2.60 \mathrm{E}-30$ & $5.15 \mathrm{E}-23$ & $1.61 \mathrm{E}-19$ \\
$0-0 \mathrm{~S}(5)$ & 6.95 & $8.02 \mathrm{E}+25$ & $1.45 \mathrm{E}+36$ & $9.87 \mathrm{E}+39$ & $1.03 \mathrm{E}-34$ & $1.87 \mathrm{E}-24$ & $1.27 \mathrm{E}-20$ \\
$1-0 \mathrm{O}(3)$ & 2.81 & $2.85 \mathrm{E}+32$ & $2.45 \mathrm{E}+37$ & $1.96 \mathrm{E}+40$ & $3.68 \mathrm{E}-28$ & $3.16 \mathrm{E}-23$ & $2.53 \mathrm{E}-20$ \\
$2-1 \mathrm{Q}(1)$ & 2.55 & $2.00 \mathrm{E}+31$ & $3.05 \mathrm{E}+36$ & $4.29 \mathrm{E}+39$ & $2.58 \mathrm{E}-29$ & $3.93 \mathrm{E}-24$ & $5.53 \mathrm{E}-21$ \\
$1-0 \mathrm{Q}(1)$ & 2.41 & $3.32 \mathrm{E}+32$ & $2.85 \mathrm{E}+37$ & $2.29 \mathrm{E}+40$ & $4.29 \mathrm{E}-28$ & $3.68 \mathrm{E}-23$ & $2.95 \mathrm{E}-20$ \\
$1-0 \mathrm{~S}(0)$ & 2.23 & $2.08 \mathrm{E}+30$ & $3.04 \mathrm{E}+36$ & $6.20 \mathrm{E}+39$ & $2.68 \mathrm{E}-30$ & $3.92 \mathrm{E}-24$ & $8.00 \mathrm{E}-21$ \\
$1-0 \mathrm{~S}(1)$ & 2.12 & $2.82 \mathrm{E}+29$ & $2.51 \mathrm{E}+36$ & $1.94 \mathrm{E}+40$ & $3.63 \mathrm{E}-31$ & $3.23 \mathrm{E}-24$ & $2.50 \mathrm{E}-20$ \\
$1-0 \mathrm{~S}(3)$ & 1.96 & $9.26 \mathrm{E}+24$ & $3.17 \mathrm{E}+34$ & $2.74 \mathrm{E}+39$ & $1.19 \mathrm{E}-35$ & $4.09 \mathrm{E}-26$ & $3.53 \mathrm{E}-21$ \\
\hline total $\mathrm{H}_{2}$ & & $4.22 \mathrm{E}+33$ & $4.89 \mathrm{E}+38$ & $4.13 \mathrm{E}+41$ & $5.44 \mathrm{E}-27$ & $6.31 \mathrm{E}-22$ & $5.33 \mathrm{E}-19$ \\
\hline H Ly $\alpha$ & & $4.74 \mathrm{E}+08$ & $3.84 \mathrm{E}+37$ & $1.87 \mathrm{E}+41$ & $6.11 \mathrm{E}-52$ & $4.95 \mathrm{E}-23$ & $2.42 \mathrm{E}-19$ \\
\hline
\end{tabular}

Table 2: The same as Table 1 but for $z=8$ 